\title{
Effective Energy Path Based PDORP Routing Protocol for WSN
}

\author{
Swapnali Latake ${ }^{1}$, Sachin V. Todkari ${ }^{2}$ \\ ${ }^{1}$ Department Computer Engineering, Jayawantrao Sawant College of Engineering, Hadapsar, Pune-28, Savitribai Phule Pune University, \\ Pune, India \\ ${ }^{2}$ Head of Department, Information Technology, Jayawantrao Sawant College of Engineering, HadapsarPune-28, Savitribai Phule Pune \\ University, Pune, India
}

\begin{abstract}
Energy consumption is the major factor in the wireless sensor network in today's era, by calculating the energy consumption any one can got to know the relevancy of the WSN. Routing protocol plays important roles to measure the quality-of-service (QoS).Key parameter in WSN for creating the overhead in the networks are many more out of these some major and some are minor parameter, minor parameter includes Energy consumption, network lifetime, network scalability and one the major parameter includes the Packet overhead. Packet overhead is the root cause of more energy consumption and reduce the QoS in sensor networks. In WSN some the routing protocol available those make sense to increase the performance in the network are Dynamic Source Routing (DSR) but the problem with such protocol is that when we move toward the big energy density it doesn't make any sense as there need to change the mode from active to sleep and vice versa. In such scenarios efficiency decreases as the data packets needs to wait at the initial point where the packet has been sent and this increases the waiting time and end to end delay of the packets which leads to increase in energy consumption. Now here need to identify that we have to find the dead nodes in between and find the new routing path so that energy consumption is get decrees and identify the good path. For such handling such scenarios we propose directional transmission based energy responsive routing protocol named as PDORP. PDORP have some best characteristics to make the routing smoother are Power Efficient Gathering Sensor Information System (PEGASIS) and DSR routing protocols. In addition to above routing protocol the genetic algorithm and Bacterial Foraging Optimization (BFO) formally used for creating and reinitiating the cluster based WSN in which it optimize the network while network establishment. The performance analysis is get calculated through the hybrid approach (soft computing techniques) of above routing protocol and off course it will gives us better result comprising less bit error rate, less delay, less energy Consumption and better quantity which clues to better QoS and extend the lifetime of the network.
\end{abstract}

Keywords: Wireless Sensor Network, Optimization Systems, Energy Consumption DSR, PEGASIS, PDORP, OD-PRRP, LEACH.

\section{Introduction}

A wireless sensor network is the network of sensor node in which one node can communicate with neighbourhood node. A wireless sensor network (WSN) typically consists of a sink node sometimes referred to as a base station and a leaf node say small sensor node. So for communication there were lots of energy required to prepared communication link, prepare the message and transfer same to Cluster node ,Sink node and then same communicate to other network [1]. So for such great communication need to have proper energy with good battery backup to each and every node in the WSN.As several cases, it is objectionable to replace the batteries that are challenging or exhausted of energy. Many scientists are in this field attempting to discover poweraware protocols for wireless sensor networks, keeping in mind the end goal to overcome such energy efficiency issues in such cases they introduces their own assumptions based on basic understanding about the WSN[2].

To optimize the WSN different scientist from different field identify and acknowledge the different design approaches [3-4]. To meet different design criteria, related researches into the optimization of WSN design can be grouped into several categories i.e. Optimization in the communication layers; Node hardware optimization and Cross-layer optimization. However, most of the optimization procedures do not take into account the principles, characteristics and requirements of WSN which is application defined. Therefore, in proposed approach energy optimization is done using hybrid algorithms i.e. GA [3] and BFO [4] method in DSR protocol.

WSN performance matrix fully dependent on network to network node communication and data transmission [5-6]. Random deployment of nodes indications many researches in the routing algorithms. Densely deployed nodes suffer from many failures due to drained battery power, environmental conditions and addition of more. Dynamic topology of WSN changes the position of the network node within the network. Such amazing features of WSN construct scalability, reliability, energy efficiency and resource management are great challenges in the design process of routing protocols. Energy consumption measure in two factor such as dynamic (mobile) and static say steady network.

To make the network load balancing by considering each node in the network. It form the network by considering the burden of each node in the network. LEACH is the one which is developed in year 2000, used the adaptive cluster approach to maximize the energy efficiency. In our case PEGASIS will be utilized frequently [7-8]. To consume nodes efficiently and sensibly is one of the important features of sensor networks. As wireless sensor nodes are prepared with non- chargeable batteries with poor energy supply, a sensor network cannot work well after a portion of the nodes run out of energy. Another challenge in front of WSN is to receive the data from reliable nodes so that are fraudulent node cant interrupt the network traffic. 


\section{International Journal of Science and Research (IJSR) \\ ISSN (Online): 2319-7064}

Index Copernicus Value (2015): 78.96 | Impact Factor (2015): 6.391

The main contributions of this paper are as follows

- It decrease the communication gap between the nodes so that less energy gets consumed and it is ensured by using directional transmission.

- To achieve shortest path, less overhead, fast response and the connectivity of the nodes are achieved by using PEGASIS routing and DSR routing methodology.

- When a node becomes more aggressive at the time of transfer and previously it was not in the cache memory, the other node is bound to receive a packet from it and in such a way it can cause damage to existing routes. A solution to this problem could be checking of any node at the time of receiving a data packet but this would cause unessential delay. Hence, the proposed solution creates a trust for the first time in each round on the basis of the parameters allocated to the nodes. After every round, the trust list is updated and after a certain number of rounds, the trust would not be checked to avoid time delays. Initialize idle listening Sensing Processing Transmission of data 2169-3536 (c) 2016 IEEE. Translations and content mining are permitted for academic research only.

- For optimized WSN, GA and BFO optimization is applied to proposed routing protocol to identify energy efficient optimal paths.

- We can see the performance of PDORP by comparing it with PEGASIS Routing Protocol (PRP).

\section{Related Work}

The paper offered by author Aarti Jain based on technique Fuzzy logic and ACO based OD-PPRP routing she identify that Clustering is one of the widely used methods to save energy, increase spatial re usability, and scalability and ODPRRP has better network lifetime, less transmission delay, high packet delivery ratio and decrease in overhead than other routing protocols like EARQ, EEABR and EAODV [9].

The paper offered by Author Xin Guan Provides more uniform energy consumption in sensor nodes and also increase the lifetime of sensor networks .To reduce the energy consumption, Pinheiro et al. propose a model for minimization of power consumption in a heterogeneous cluster of computing nodes serving multiple webapplications, which periodically monitors the load of resources and makes decisions on switching nodes on/off to minimize the overall power consumption [10-11].

The paper offered by author Akimitsu Kanzaki, Yasuhiro Nose introduce that we can construct effective communication routes in terms of both power consumption and the quality of communication. They proposed the method for finding or managing the route in WSN for communication and network formation in WSN [12].

The paper offered by author Young based on technique a cross-Layer Channel Access and Routing Protocol in that he introduces a new reliable protocol termed Cross-layer Channel Access and Routing (CCAR), which simultaneously supports both MAC and routing operations for medical-grade QoS provisions. It initially defines the routing path with the lowest traffic load and low latency using newly defined channel quality factors. Concurrently, the source node allocates the predefined QoS Access Category to each packet and reserves the channel by considering the route. CCAR introduces an effective route maintenance scheme to avoid link failures in bottlenecked intermediate nodes, which prevents unnecessary packet drops and route rediscovery evocations. Finally, through both simulation studies and real test-bed experiments, we evaluate the performance of CCAR by comparing it with other conventional protocols, demonstrating that the proposed protocol can more efficiently support medicalgrade QoS packets, especially when the network is heavily loaded [13].

\section{Proposed Work}

We suggest a scheme, named ENERGY EFFICIENT DIRECTION BASED PDORP ROUTING PROTOCOL FOR WSNscenarios In that we propose directional transmission based energy responsive routing protocol named as PDORP. PDORP have some best characteristics to make the routing smoother are Power Efficient Gathering Sensor Information System (PEGASIS) and DSR routing protocols. In addition to above routing protocol the genetic algorithm and Bacterial Foraging Optimization (BFO) formally used for creating and reinitiating the cluster based WSN in which it optimize the network while network establishment. The performance analysis is get calculated through the hybrid approach (soft computing techniques) of above routing protocol and off course it will gives us better result comprising less bit error rate, less delay, less energy Consumption and better quantity which clues to better QoS and extend the lifetime of the network.

Proposed system will go through following flow of execution,

1) Network creation (WSN formation) algorithm.

2) Path finding algorithm.

3) Routing Cache DSR Integration (PDORP) algorithm.

4) Hybrid Algorithm (Action of GA and BFO) algorithm.

\section{Architectural View}

The architecture diagram of the system shown below helps us to understand the system.

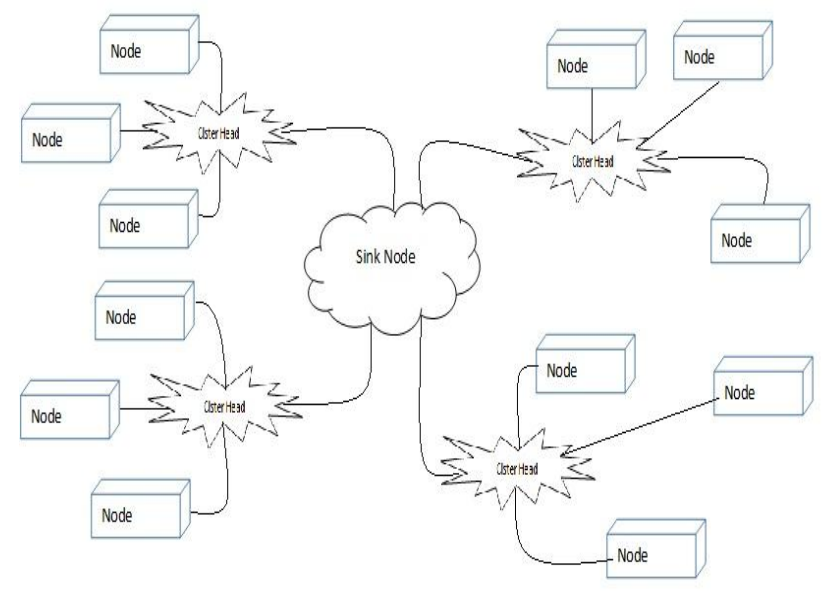

Figure: System Architecture 


\section{International Journal of Science and Research (IJSR) \\ ISSN (Online): 2319-7064 \\ Index Copernicus Value (2015): 78.96 | Impact Factor (2015): 6.391}

In above System architecture diagram each leaf node can communicate with his cluster head and then cluster head can communicate with sink node. So our agenda is to find minimum path required for communication by analysing performance analysis to reach to the sink node say base station. In proposed system we get QoS by analysing our system and previous system and shown using simulation kind of application.

\begin{tabular}{|c|c|c|c|c|}
\hline $\begin{array}{l}\mathrm{Sr} \\
\text { No. }\end{array}$ & Paper & Technique & Advantages & Disadvantage \\
\hline 1 & $\begin{array}{c}\text { A Novel Method of Modeling } \\
\text { Wireless Sensor Network Using } \\
\text { Fuzzy Graph and Energy Efficient } \\
\text { Fuzzy Based k-Hop Clustering } \\
\text { Algorithm. }\end{array}$ & $\begin{array}{l}\text { Fuzzy logic and } \\
\text { ACO based } \\
\text { OD-PPRP } \\
\text { routing. }\end{array}$ & $\begin{array}{l}\text { OD-PRRP has better network lifetime, } \\
\text { less transmission delay, high packet } \\
\text { delivery ratio and decrease in overhead } \\
\text { than other routing protocols like EARQ, } \\
\text { EEABR and EAODV. }\end{array}$ & $\begin{array}{l}\text { It is applicable only on WSN } \\
\text { network, so can be applied to } \\
\text { MANET or wireless network. }\end{array}$ \\
\hline 2 & $\begin{array}{l}\text { Job Scheduling Model for Cloud } \\
\text { Computing Based on Multi- } \\
\text { Objective Genetic Algorithm. }\end{array}$ & $\begin{array}{l}\text { Load balance } \\
\text { data gathering } \\
\text { algorithm. }\end{array}$ & $\begin{array}{c}\text { Provides more uniform energy } \\
\text { consumption in sensor nodes and also } \\
\text { increase the lifetime of sensor networks. }\end{array}$ & $\begin{array}{l}\text { Further extension of this protocol } \\
\text { and make it adapt to the three- } \\
\text { dimensional Environment. }\end{array}$ \\
\hline 3 & $\begin{array}{c}\text { Dynamic Route Construction Based } \\
\text { on Measured Characteristics of } \\
\text { Radio Propagation in Wireless } \\
\text { Sensor Networks. }\end{array}$ & $\begin{array}{c}\text { Error correcting } \\
\text { technique }\end{array}$ & $\begin{array}{l}\text { Can construct effective communication } \\
\text { routes in terms of both power } \\
\text { consumption and the quality of } \\
\text { communication. }\end{array}$ & $\begin{array}{l}\text { Some metrics other than the } \\
\text { packet delivery ratio seem to be } \\
\text { needed to further improve the } \\
\text { quality of communication. }\end{array}$ \\
\hline 4 & $\begin{array}{c}\text { A Cross-Layer Channel Access and } \\
\text { Routing Protocol for Medical- } \\
\text { Grade QoS Support in Wireless } \\
\text { Sensor Networks. } \\
\end{array}$ & $\begin{array}{l}\text { A cross-Layer } \\
\text { Channel Access } \\
\text { and Routing } \\
\text { Protocol. } \\
\end{array}$ & $\begin{array}{l}\text { The proposed protocol can more } \\
\text { efficiently support QoS packets, even the } \\
\text { network is highly loaded. }\end{array}$ & $\begin{array}{c}\text { Need to investigate more detailed } \\
\text { QoS requirements of medical } \\
\text { information systems. }\end{array}$ \\
\hline 5 & $\begin{array}{l}\text { Advanced verification on WBAN } \\
\text { and cloud computing for u-health } \\
\text { environment. }\end{array}$ & $\begin{array}{l}\text { Integration } \\
\text { platform based } \\
\text { on WBAN. }\end{array}$ & $\begin{array}{l}\text { Optimized the WBAN and the TCP as a } \\
\text { sampling rate control. }\end{array}$ & $\begin{array}{c}\text { Usage of Zigbee protocols } \\
\text { because it has a slower power } \\
\text { consumption rate. }\end{array}$ \\
\hline 6 & $\begin{array}{l}\text { "Predictive routing for mobile sinks } \\
\text { in wireless sensor networks: a } \\
\text { milestone based approach. }\end{array}$ & $\begin{array}{l}\text { Milestone- } \\
\text { based predictive } \\
\text { routing }\end{array}$ & $\begin{array}{l}\text { The proposed routing protocol reduces } \\
\text { energy consumption and packet delivery } \\
\text { ratios in comparison to previous routing } \\
\text { protocols such as ALURP and Elastic. }\end{array}$ & $\begin{array}{c}\text { Improve the predictive protocol } \\
\text { by managing QoS in sink } \\
\text { mobility WSNs. }\end{array}$ \\
\hline
\end{tabular}

In our proposed system PDORP, both the proactive routing and reactive routing methodology have been used in order to obtain fast and non-damaged path along with lower transmission delay in WSN will show through comparison simulation while creating above type of WSN.

\section{Conclusion}

In proposed system, we offered hybrid optimization based PEGASIS-DSR optimized routing protocol (PDORP), which includes cache and directional transmission concept for both proactive and reactive routing protocols. The simulation results of our proposed system show reduction in end to end transmission delay and bit error rate without compromising with energy efficiency. In PDORP, both the proactive routing and reactive routing methodology have been used in order to obtain fast and non-damaged path along with lower transmission delay. The performance of DORP has been evaluated by comparing with existing available methods.

\section{References}

[1] M. Merck."The icecube detector: A large sensor network at the south pole". IEEE Pervasive Computing, 2010,vol. (4), pp 43-47.

[2] T.H. Feiroz Khan, D. Sivakumar, "Performance of AODV, DSDV and DSR protocols in Mobile Wireless Mesh Networks", IEEE 2nd International Conference on Current Trends in Engineering and Technology,2014, Coiambtore ,pp 397-399.

[3] Mohammed Abo-Zahhad, Sabah M. Ahmed, Nabil Sabor and Shigenobu Sasaki, "A New Energy Efficient Adaptive Clustering Protocol Based on Genetic Algorithm for Improving the Lifetime and the Stable
Period of Wireless Sensor Networks", International Journal of Energy, Information and Communications Vol.5, Issue 3 (2014).

[4] Chen Han-ning, Zhu Yun-long, $\mathrm{Hu}$ Kun-yuan, "Cooperative bacterial foraging algorithm for global optimization", IEEE Chinese control and decision conference, Guilin, China, 2009, vol. 6,pp 3896-3901.

[5] L.G. Quesada. "A Routing Protocol for MANETs", In Master of Science in Communication Technology thesis,

2007,(http://brage.bibsys.no/xmlui/handle/11250/26191 $6)$.

[6] H. AlAamri, M. Abolhasan, and T. Wysocki, On Optimising "Route Discovery in Absence of Previous Route Information in MANETs", Proc. IEEE Vehicular Technology Conference (VTC), Barcelona, 2009,pp. 15.

[7] H. Shen, Y. L. Zhu, X. M. Zhou, H. F. Guo, C. G. Chang, "Bacterial foraging optimization algorithm with particle swarm optimization strategy for global numerical optimization", World Summit on Genetic and Evolutionary Computation, 2009, Shanghai, China, pp. 497-504.

[8] Kou Pan-gao, Zhou Jian-zhong, “Optimal PID Governor Tuning of Hydraulic Turbine Generators With Bacterial Foraging Particle Swarm Optimization Algorithm". In Proceedings of the CSEE, 2009, Vol. 29(26), pp 101-106.

[9] Aarti Jain and B. V. Ramana Reddy.“A Novel Method of Modeling Wireless Sensor Network Using Fuzzy Graph and Energy Efficient Fuzzy Based k-Hop Clustering Algorithm". Wireless Personal Communications. 2015, vol 82( 1), pp. 157-181.

\section{Volume 6 Issue 1, January 2017




\section{International Journal of Science and Research (IJSR) \\ ISSN (Online): 2319-7064}

Index Copernicus Value (2015): 78.96 | Impact Factor (2015): 6.391

[10] Jing Liu, Xing-Guo Luo, Xing-Ming Zhang, Fan Zhang and Bai-Nan Li, "Job Scheduling Model for Cloud Computing Based on Multi-Objective Genetic Algorithm", IJCSI International Journal of Computer Science Issues, Vol. 10, Issue 1, No 3, January 2013, pp 134-139

[11]E. Pinheiro, R. Bianchini, E.V. Carrera and T. Heath, "Load balancing and unbalancing for power and performance in cluster-based systems", in Proceedings of the Workshop on Compilers and Operating Systems for Low Power, 2001, pp. 182-195.

[12] Akimitsu Kanzaki, Yasuhiro Nose, Takahiro Hara, \& Shojiro Nishio, "Dynamic Route Construction Based on Measured Characteristics of Radio Propagation in Wireless Sensor Networks", International Journal of Advanced Computer Science, vol. 2, No. 3, Pp. 85-98, Mar. 2012.

[13] Young-Duk Kim, Kook-Rae Cho, Hui-Sup Cho, and Dongkyun Kim. 2014. “A Cross-Layer Channel Access and Routing Protocol for Medical-Grade QoS Support in Wireless Sensor Networks". Wireless Personal Communications,2014, vol. 77( 1), pp 309-328.

\section{Author Profile}

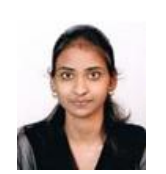

Miss. Swapnali Latake is currently pursuing M.E (Computer) from Department of Computer Engineering, Jayawantrao Sawant College of Engineering, Pune, India. Savitribai Phule Pune University, Pune, Maharashtra, India -411007. She received her B.E (Computer) Degree from D. Y. Patil College of Engineering, Kolhapur, India. Shivaji University, Kolhapur, Maharashtra, India. Her area of interest is network security, WSN.

Prof. S. V. Todkari Received his M.E (Information Technology) from Savitribai Phule Pune University, Pune, Maharashtra, India 411007. He received his B.E (Computer) Degree from College of Engineering, Ambejogai, India. BAMU, Maharashtra, India. His area of interest is network security, WSN. 